\title{
Development of an Automatic Transportation System Intended for Long Tunnels
}

\author{
Hitoshi Suzuki*, Toshifumi Umegae*, Kuniaki Fujita*, Satoru Kozuki*, \\ Satoru Saito*, Hiroshi Takahashi**, and Yuji Murakami** \\ * Sato Kogyo Co., Ltd., Miyagase field office, Yokohama Branch, 1710 Toriibara, Toya, \\ Tsukui-machi, Tsukui-gun, Kanagawa Pref. 220-02, JAPAN \\ ** Sato Kogyo Co., Ltd., Civil Engineering Div., Engineering Dept., 4-12-20 Nihonbashi- \\ honcho, Chuo-ku, Tokyo 103, JAPAN
}

\begin{abstract}
With the rapid advancement of tunnel-boring machines, driving operation has recently been carried out with a higher level of speed and efficiency. However, in the case of long tunnels, a rapid transportation system that supports speedy driving is indispensable. It is also important, after carrying out accurate monitoring of a flow of work involved in tunnel driving, from workers' going-in and -out of the tunnel, construction equipment and materials supply, to spoil removal operation, to maintain proper control of the entire work process to achieve the smooth progress of tunnel driving with enhanced safety. In order to achieve this, and to ensure the safety of workers during the process of rapid transportation, automating tunnel haulage operation and thus minimizing the number of operators and workers inside the tunnel is a viable strategy. A system developed to fulfill these requirements comprises more than one unattended train that travel back and forth inside the tunnel and a train operation control device that continuously monitors on-going operations of the trains to ensure safety. This paper gives an overview of the "automatic transportation system intended for long tunnels" and reports the application of this system to Doushi Headrace (Hayato section) construction.
\end{abstract}

\section{INTRODUCTION}

Located in a mountainous area of Kanagawa Prefecture, just $50 \mathrm{~km}$ from the centre of Tokyo in Japan, the $156 \mathrm{~m}$ high Miyagase dam will provide fresh reservoir capacity of 193 million $\mathrm{m}^{3}$. The multiple purposes catered for in its design are: to control flooding in the area of the Nakatsu river; maintain the normal functions of the flowing water of the main streams of both the Nakatsu and the Sagami rivers; reinforce regional water supplies in Kanagawa Prefecture; and generate $24,200 \mathrm{~kW}$ of electricity at a new hydro plant to serve 25,000 homes. The owner of the new dam is the Ministry of Construction.

The water resources of the area are already harnessed by the Sagami and Shiroyama dams. Both these dams have such a small capacity that the water resource cannot be fully and effectively utilised, but integrated operation with the new Miyagase dam of larger capacity 


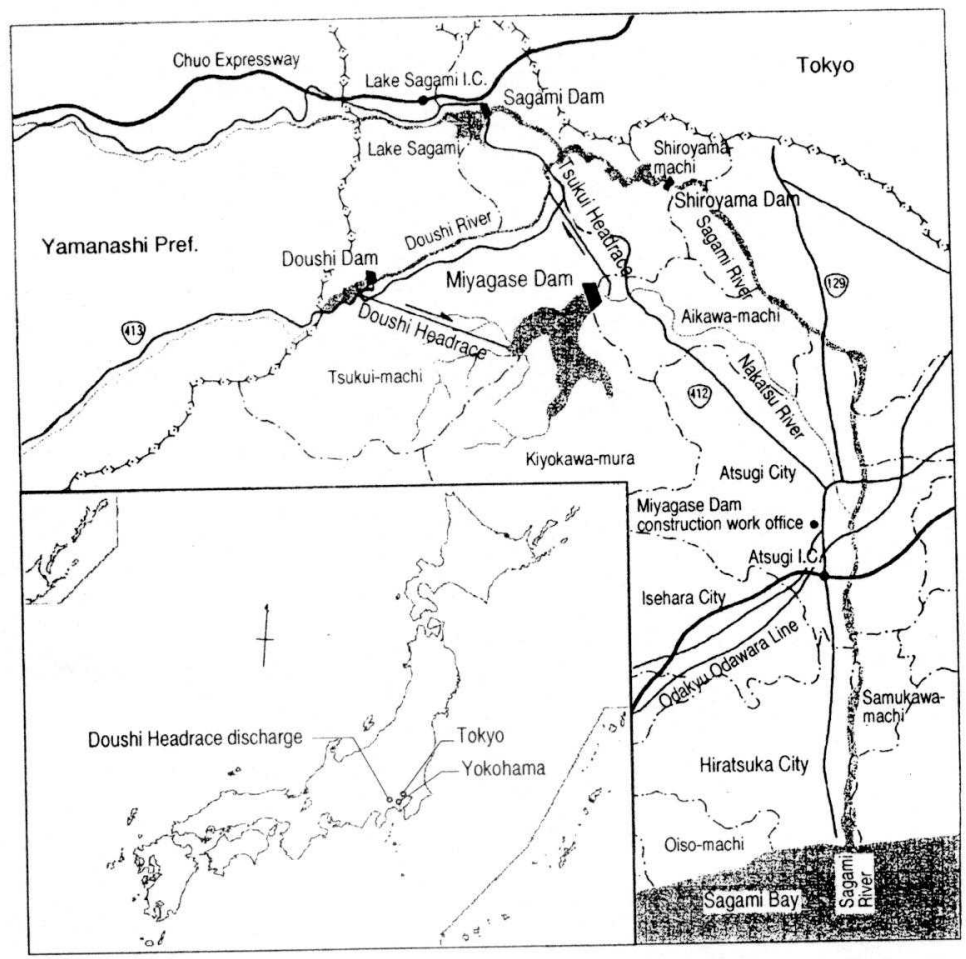

Figure 1 Location map

will rectify this situation. To achieve this, an $8 \mathrm{~km}$ headrace tunnel is being driven from the Doushi dam to Mayagase and a second $5 \mathrm{~km}$ headrace tunnel is under construction from Miyagase to the Doushi river.

\section{OUTLINE OF THE CONSTRUCTION}

The Doushi headrace tunnel is being constructed by Sato Kogyo JV using a fully-shielded $3.5 \mathrm{~m}$ diameter Kawasaki hardrock TBM. It is being driven in a westerly direction from a leg of the proposed Miyagase lake at $0.273 \%$ grade towards Doushi through five major and sixteen minor faults. Most of the alignment is in tuff varying in uniaxial compressive strength from 40 to $180 \mathrm{MPa}$, with a central section of about $1 \mathrm{~km}$ in andesite of 100 to $200 \mathrm{MPa}$. Two major zones comprising basalt lava and diorite will also be met in the later stages of the drive.

There are two $300 \mathrm{~m}$ radius bends, one near the intake portal and the other near the discharge portal, and the remainder of the drive is in a straight line.

A $150 \mathrm{~mm}$ thick, rubber-gasketed reinforced concrete segmental lining is installed by a rear-mounted erector within the tailskin of the TBM. The annulus is filled three rings back from the erector using $1 \mathrm{~m}^{3} /$ ring cemented grout pumped onto the job along a pipeline from surface. Each $1 \mathrm{~m}$ ring comprises four segments and a key segment, and the finished internal diameter is $3.0 \mathrm{~m}$. Segment manufacture is spread over three different suppliers and a stock of around sixty rings is maintained at site, where a $4 \mathrm{t}$ pedestal crane handles the loading.

The first $54 \mathrm{~m}$ from the portal was driven by drill/blast using steel arches and shotcrete for support. The TBM and its trailing backup were first assembled outside the tunnel and then hauled into the launch position. 


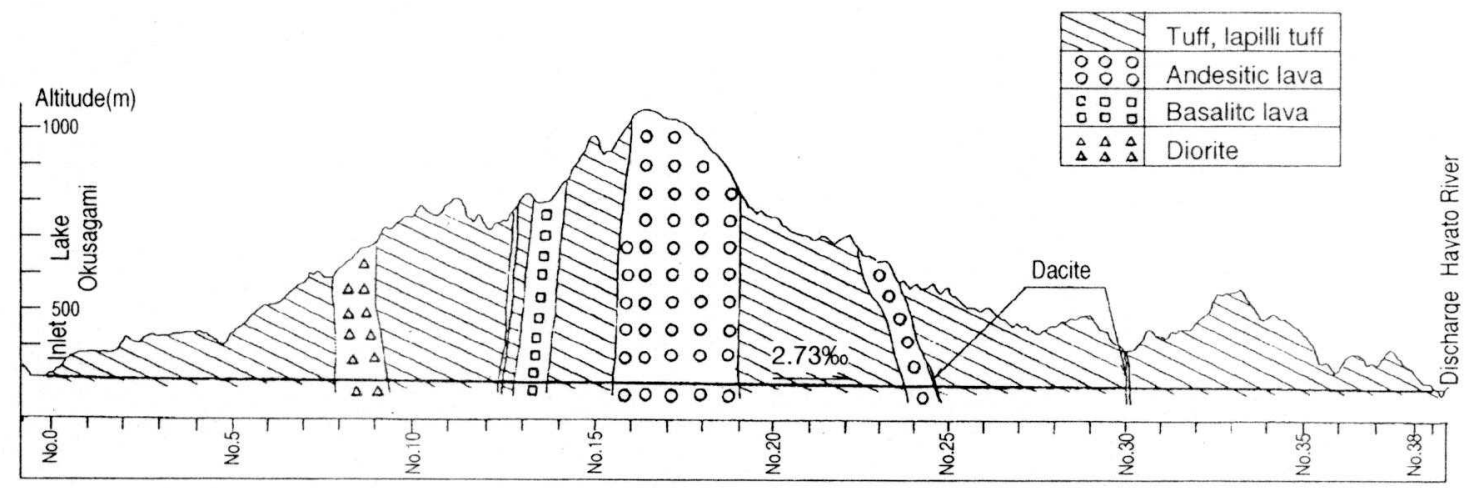

Figure 2 Geological profile

Boring commenced at the end of June, 1994 using two $10 \mathrm{~h}$ shifts/day on five days/week for a planned advance of $260 \mathrm{~m} / \mathrm{month}$.

\section{CHARACTERISTIC FEATURES OF THE SYSTEM}

The Doushi Headrace tunnel is being driven with a tunnel boring machine (TBM), followed by segmental lining installation. The finished internal diameter is as small as $3.0 \mathrm{~m}$. In the case of attended mine car operation, fourteen operators are necessary, and it takes them more than one hour to go in and out of the tunnel. From the points of view of achieving enhanced safety and minimizing the number of operators, the system configuration was designed taking into account such elements as the traveling speed of the trains, the number of trains operated, and the number of blocks in which trains pass each other (passing blocks). Given below are the characteristic features of the system.

(1) Unmanned and safety operation of a max. distance of $7 \mathrm{~km}$ and five trains (max.) is possible.

1) Proper distance can be maintained between the trains throughout the operation.

2) All trains are mounted with various types of sensors (obstruction detector, obstruction detecting bumper) to ensure safety.

(2) Material supply that occurs on an irregular basis can be coped with by the operation of the attended train between scheduled unattended train services.

1) To ensure safety operation of the attended train, signals are controlled by continuous monitoring and controlling of the position of each train.

2) The attended train is also kept under control and monitoring. As soon as the attended train is found to be a hindrance to the operation of the unattended train (e.g. disregard of signals, unexpectedly long resident time inside the sections shared by the attended and unattended trains, etc.), the operation of all the unattended trains will be suspended. 
(3) Feedback of the analytical results of operational records to the on-going train operation helps improve the safety and efficiency of the tunnel driving work.

1) Mechanical conditions of the trains are grasped from the emergency stop records of all trains to perform precautionary maintenance.

2) Waiting hours consumed in the passing blocks are analyzed. The results will be used in, discussing the proper location of subsequent passing blocks and traveling speed levels.

\section{OVERVIEW OF THE SYSTEM}

\subsection{AUTOMATIC OPERATION OF THE UNATTENDED TRAINS}

The system in question was developed to allow the automatic operation of the unattended trains used both for segment supply and tunnel spoil removal. The system is remotely controlled by a central control device installed outside the tunnel. The trains travelling on a single track pass each other in the passing blocks set up at several locations along the route. Any one section sandwiched between two adjacent passing blocks is regarded as a unit operation block, and the operation of each train is controlled automatically using this block as a minimum traveling unit. All the trains are required to stop at the entrance to each passing block before proceeding to the block immediately ahead. The trains iterate this process at the entrance to each passing block. The trains can proceed to the subsequent block only when the following requirements are fulfilled.

(1) Two trains, up- and down-tracks each, are concurrently present within the passing block.

(2) No train traveling in the same direction is present within the two unit operation blocks immediately ahead.

(3) No oncoming train is present within the unit operation block immediately ahead.

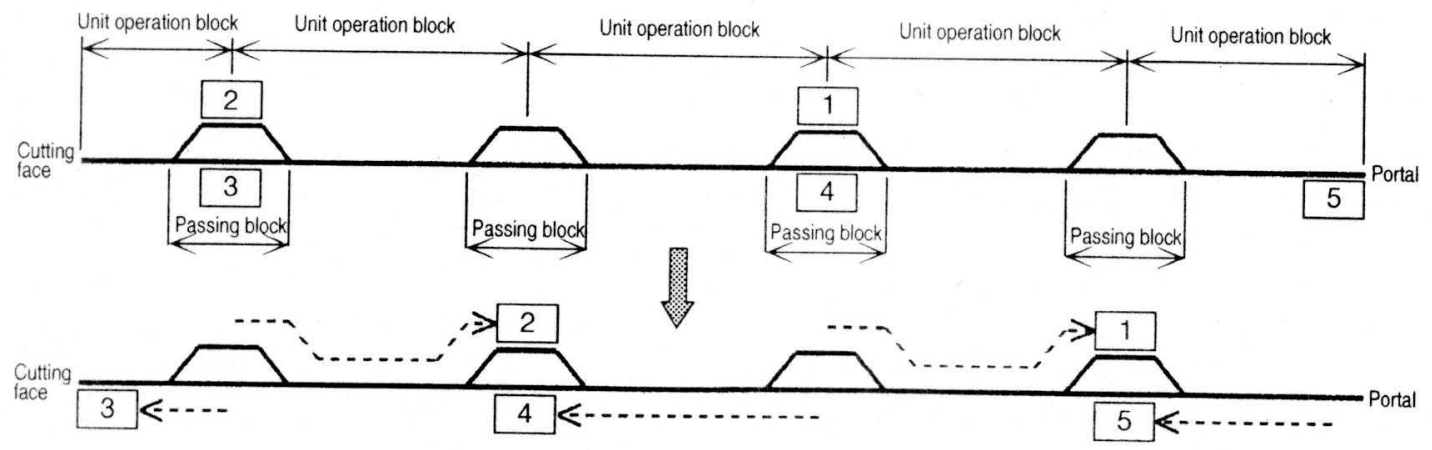

Figure 3 Automatic operation regulations 


\subsection{OPERATION OF THE ATTENDED TRAIN}

As mentioned in the previous section, the use of system is twofold: segment supply and spoil removal. The attended train is used as a means of access for the workers to the tunnel, as well as for irregular supply of backfill materials. The attended and unattended trains share the track within the passing blocks (Figure 4). Based on an unattended-cars-comes-first principle, following control items are being exercised:

(1) The central control device monitors the position of each and every train, and controls signals to ensure the smooth and safe advancement of the attended train into the shared sections.

(2) The attended train is placed under continuous monitoring. As soon as it is found to be a hindrance to the operation of the unattended trains (e.g. disregard of signals, unexpectedly long resident time inside the sections shared by the attended and unattended trains, etc.), the operation of the all the unattended trains will be suspended.

\section{SYSTEM COMPONENTS}

Major components of the system are given below. System configuration is presented in Figure 5.

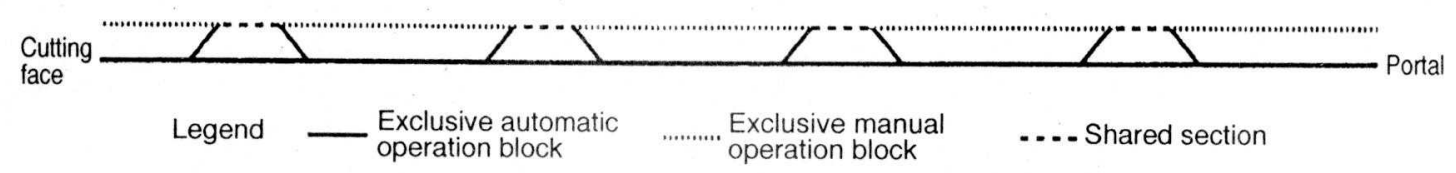

Figure 4 Conceptual drawing of the track shared by attended and unattended trains

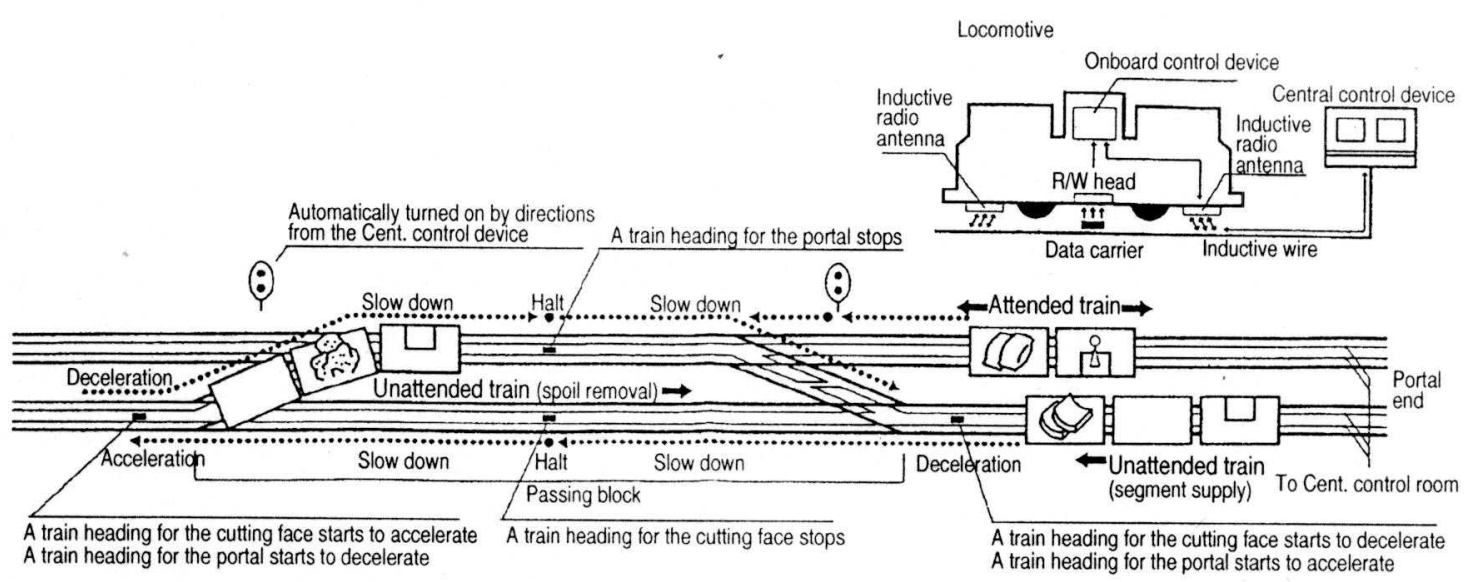

Figure 5 System configuration 
(1) Central control device

The central control device installed in the central control room located outside the tunnel collects real-time data from various types of devices, based on which, control of automatic train operation is performed. The central control device also outputs operation status data and issues alarm signals.

(2) Inductive radio system

The inductive radio system allows the central control device to issue instructions to the trains by radio communications. It also allows the trains to transmit operational data to the central control device.

(3) Unattended trains

The unattended trains are automatically operated by instructions issued via the inductive radio system from the central control device as well as by data written on ID tags set up along the track. The unattended trains are loaded with safety devices and alarm devices.

(4) Attended train

The attended train continuously sends out position data to the central control device via the inductive radio system. According to the data received, the central control device issues signals to control operations of the attended train that is run on an irregular basis.

(5) ID tags

The ID tags installed along the track carry data necessary for train operations. The ID tags continuously transmit information to passing trains.

\section{SYSTEM CONTROL}

System control is effected by data exchange between the central control device installed outside the tunnel and programmable controllers mounted on the trains. The ID tags installed at primary points along the track continuously transmit information containing the number of the current train position, the distance from the portal, and proper traveling speed levels that vary according to traveling direction. The trains read out as they pass by these tags necessary information via $\mathrm{R} / \mathrm{W}$ heads.

The trains compute travel distance using built-in encoders and distance data and transmit a set of real-time data comprising current position, traveling direction and velocity, destination (point number), etc. to the central control device. Having grasped the traveling conditions of all the trains using the data received from the trains, the central control device issues necessary instructions to each train (destination, emergency stop, etc.).

The sensors mounted on the trains detect abnormal operating conditions, such as troubles and defects the trains develop, the presence of obstructions on the rail, and overrunning of the trains beyond the system control section. As soon as these abnormalities are detected, the trains are automatically brought to a halt and transmit necessary information to the central control device.

The on-going state of operations are displayed on the monitor screen set up in the central control room and TBM operation room. Accumulated operational data sent from individual trains are processed into statistical data to be used during review of operational planning and precautionary maintenance. 


\section{APPLICATION OF THE SYSTEM}

Figure 6 illustrates the track and its auxiliary equipment installed inside the tunnel and Figure 7, cross sections.

(1) Passing blocks (fixed point)

The passing blocks are provided at $2250 \mathrm{~m}$ intervals.

(2) Advanced passing block (movable)

In order to ensure smooth shunting of a segment supply train and a muck train, the advanced passing block is provided at the rear of carriages attached to the TBM. The advanced passing block is moved forward as the driving progresses.

(3) Single-track blocks (fixed)

The single-track blocks are provided at $1400 \mathrm{~m}$ intervals for transformer and relay pump installation.

The passing and single-track blocks have particular sections shared by the unattended and attended trains.

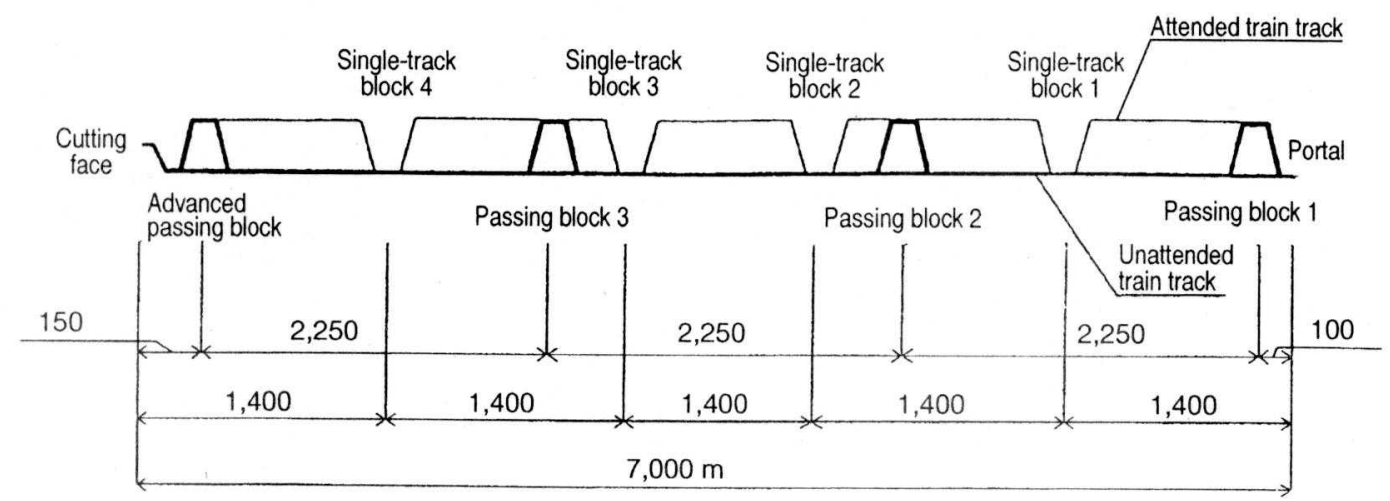

Figure 6 Track planning when the max. driving distance $(7000 \mathrm{~m})$ is reached

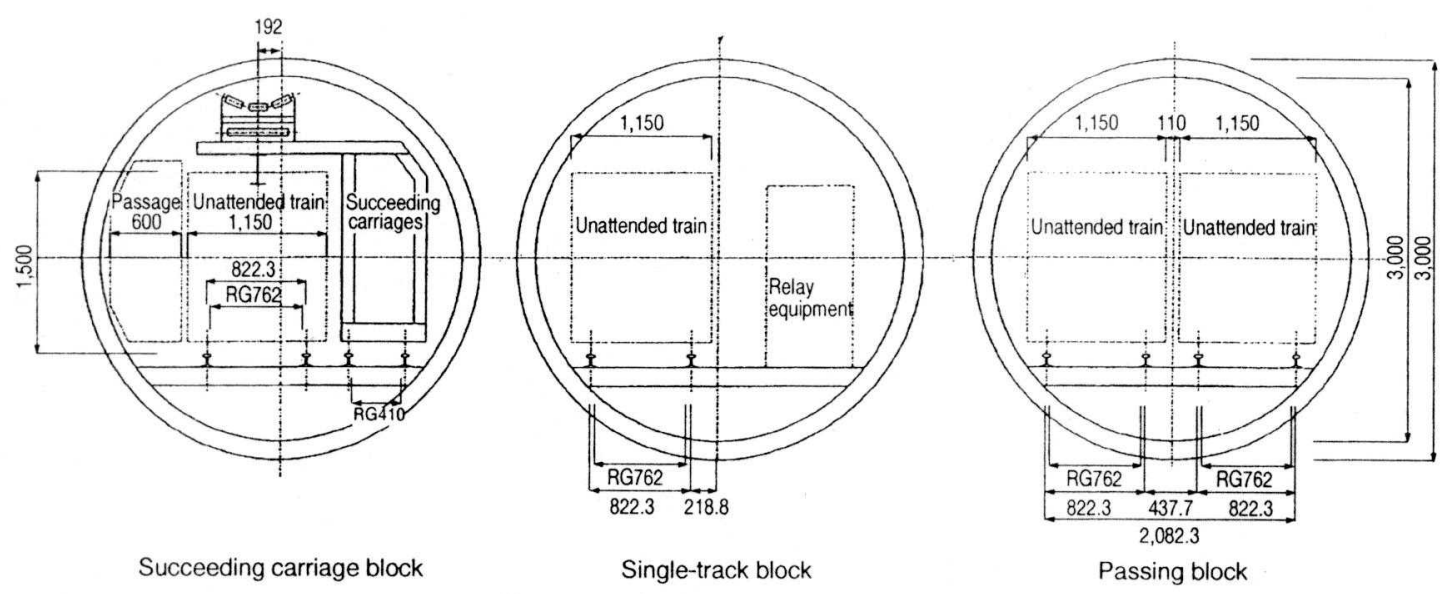

Figure 7 Tunnel cross sections 
At the tunnel driving site, the segment supply train and the muck train run back and forth on a track exclusively used by the unattended trains (on the left) at a travelling speed of 14 $\mathrm{km} / \mathrm{h}$ and they pass each other (at $5 \mathrm{~km} / \mathrm{h}$ ) within the passing block. The irregularly operated attended train travels on a track for use exclusively by the attended train (on the right) at a speed of $12 \mathrm{~km} / \mathrm{h}$. Signals are installed in the shared sections, and the attended train travels following the signal directions. Disregard of signals or violation of the rules and regulations results in the suspension of the operation of all the unattended trains.

The system was introduced for the first time in the tunnel driving site in September 1994. The total length excavated up until the end of October 1995 is $4300 \mathrm{~m}$. The system comprising four unattended trains and one attended train is currently in service without troubles. A monthly advance of $409 \mathrm{~m}$ was recorded in November 1994, a daily advance of $28 \mathrm{~m}$ on May 30, 1995 and a monthly advance of $402 \mathrm{~m}$ in June 1995, contributing greatly to speedy tunnel construction.

A major problem at this point lies in the inductive radio system. Because of the short communication distance, a pair of inductive wires are currently installed $30 \mathrm{~cm}$ apart. The problem here is that wiring is time-consuming and, in addition, parallel installation is impossible in the switch point section, causing poor communication conditions.

\section{AFTERWORD}

The system is highly estimated because of its enhanced safety, labor-saving effect, and improved work efficiency. By accumulating data to be obtained from future applications, the authors wish to realize a further developed version of the current system that features a wellbalanced combination of economy, safety and productivity.

\section{REFERENCES}

1) Takahashi, et al., 1995. "Development of a long distance tunnel transportation system (Part 1), -Functions and characteristics of the system-", The Japan Society of Civil Engineers' 50th science lecture meeting.

2) Suzuki, et.al., 1995. "Development of a long distance tunnel transportation system (Part 1), -Application of the system to Doushi Tunnel construction-", The Japan Society of Civil Engineers' 50th science lecture meeting.

3) Kouzuki, et al., 1995. "New technologies for rapid tunnel driving using TBM", The 36th meeting for presenting practical experience of tunnel construction, -Automation of tunnel driving work-.

4) World Tunneling, April 1995. 